\title{
Arthroscopic Bankart Repair vs. Open Latarjet Procedure: A Comparative Study of Clinical and Functional Results.
}

\section{Binod Sherchan}

National Trauma Center, National Academy of Medical Sciences, Kathmandu, Nepal

\section{Saroj Rai ( $D$ mesaroz@outlook.com )}

National Trauma Center, National Academy of Medical Sciences https://orcid.org/0000-0001-68963928

\section{Nira Tamang}

Norvic International Hospital, Kathmandu

\section{Siddhartha Dhungana}

National Trauma Center, National Academy of Medical Sciences, Kathmandu

\section{Laxmi Kanta Sharma}

National Trauma Center, National Academy of Medical Sciences, Kathmandu

\section{Rudra Prasad Marasini}

National Trauma Center, National Academy of Medical Sciences, Kathmandu

Janith Lal Singh

National Trauma Center, National Academy of Medical Sciences, Kathmandu

Kiran Khanal

National Trauma Center, National Academy of Medical Sciences, Kathmandu

\section{Research article}

Keywords: Arthroscopy, Bankart repair, Laterjet procedure

Posted Date: June 23rd, 2020

DOl: https://doi.org/10.21203/rs.3.rs-36217/v1

License: (c) (i) This work is licensed under a Creative Commons Attribution 4.0 International License.

Read Full License 


\section{Abstract}

Background The primary aim of this study was to compare the clinical outcomes of patients undergoing arthroscopic Bankart repair and open Latarjet procedure for recurrent dislocation of the shoulder. Secondary aims were to assess and compare the cost-effectiveness, satisfaction and complications, including recurrence and infection.

Methods We retrospectively evaluated the patients who underwent either arthroscopic Bankart repair with or without Remplissage procedure or open Latarjet procedure between May 2015 and May 2018. The preoperative data were collected from the hospital records, and the postoperative data were collected during the follow-up visit. At the final follow-up, 41 (male=32, female=9) patients in the Bankart group and 40 (male=34 and female=6) patients in the Latarjet group were included in the study. Clinical outcomes were assessed using the ASES score, Rowe score, and Quick DASH score. A self-constructed scale that consisted of satisfied and dissatisfied was used to measure the level of satisfaction. Any complications were recorded in every follow-up visit. Collected data were analyzed using SPSS version 25.

Results There was no statistically significant difference regarding the age $(p=0.401)$, gender $(p=0.569)$, site of involvement $(p=0.158)$, number of preoperative dislocations $(p=0.085)$, follow-up $(p=0.061)$, between the two groups. Similarly, no statistically significant difference existed regarding the ASES score $(p=0.388)$, Rowe score $(p=0.211)$, and Quick DASH score $(p=0.713)$. The average external rotation was 83 degrees in the Bankart group and 85 degrees in the Latarjet group $(p=0.140)$. Functional satisfaction was higher in the Laterjet group compared to the Bankart group $(p=0.482)$. Hundred percent of the patients were cosmetically satisfied in the Bankart group, whereas only $32(80 \%)$ patients were cosmetically satisfied in the Latarjet group $(p=0.002)$. There was a significantly higher operating cost for arthroscopic Bankart repair compared to open Latarjet procedure $(p<0.001)$. Three patients had a recurrence in the Bankart group, whereas no recurrence occurred in the Latarjet group. And, 2 superficial infections occurred in the Latarjet group.

Conclusion These results provide the fact that arthroscopic Bankart repair might be a fancy and minimally invasive procedure, Latarjet procedure should still be a priority in a developing country like Nepal, where financial cost is a huge burden.

\section{Background}

Anterior shoulder dislocation is one of the most common dislocations among all the joints, and occurs in $2 \%$ of the population with an overall incidence rate of 23.9 per 100,000 per year $[1,2]$. The incidence of recurrent dislocation is higher if the first dislocation occurs in less than 20 years of age [3]. Surgical stabilization is only the viable option to deal with recurrent shoulder dislocation that provides a robust fixation as nonoperative treatment is reserved for only those who are unable to tolerate surgery, as it is associated with a high rate of recurrence even in the first time dislocator $[4,5]$. 
The choice of surgical procedure is usually dependent upon the surgeon's expertise and preferences, demand for postoperative activities, and critical bone loss [6, 7]. The critical bone loss is defined as the glenoid bone loss, which significantly reduces the stability of the shoulder, which ranges from $13 \%-25 \%$ [8-14].

Arthroscopic Bankart repair using the suture anchors is a minimally invasive treatment method [13]. General indication of arthroscopic Bankart repair is soft tissue Bankart lesion with or without minimal bone loss. An additional Remplissage procedure is indicated for patients with a large $(>25 \%)$ engaging Hill-Sach's defect $[15,16]$ In contrast, the open Latarjet procedure is regarded as a gold-standard method and is being performed for decades. General indication of Latarjet procedure is recurrent shoulder dislocation with a significant glenoid bone loss. Both of these surgical techniques have their merits and demerits, which will be debated for years to come; however, surgical satisfaction depends on the clinical outcomes and the recurrence $[5,6,12-14,17-20]$.

We considered a critical glenoid bone loss as $20 \%$. We generally chose arthroscopic Bankart repair for patients with soft tissue Bankart lesion or glenoid bone loss of $<20 \%$. Remplissage procedure is added if Hill-Sach's defect is significantly large $(>25 \%)$. The open Latarjet procedure is considered if the patients have $20 \%$ or more glenoid bone loss or significant Hill Sach's lesion. However, in a developing country like Nepal, the patients' financial factors have to be considered for the choice of surgical procedures. Many of our patients are unable to afford expensive suture anchors for arthroscopic Bankart repair as most of them do not have basic medical insurance, and even if they have, the cost of the implants is not covered by the insurance $[21,22]$.

We divided the patients into the Bankart group and Latarjet group according to the surgical procedure they underwent. The primary aim of this study was to compare the clinical outcomes of patients undergoing arthroscopic Bankart repair and open Latarjet procedure for recurrent dislocation of the shoulder. Secondary aims were to assess and compare the cost-effectiveness, satisfaction and complications, including recurrence and infection.

\section{Methods}

\section{Patients}

We retrospectively evaluated the patients who underwent either arthroscopic Bankart repair with or without Remplissage procedure or open Latarjet procedure between May 2015 and May 2018. The preoperative data, including patients' demographic details, date of surgery, types of surgical procedure, and any perioperative complications, were collected from the hospital records. Postoperative data, including clinical outcomes and complications, were collected during the follow-up visit.

At the final follow-up, 41 (male=32, female=9) patients in the Bankart group and 40 (male=34 and female=6) patients in the Latarjet group were included in the study. The inclusion criteria for the study were (1). Patients undergoing either arthroscopic Bankart repair with or without Remplissage procedure or 
open Latarjet procedure with a minimum follow-up of 2 years, and (2). All patients having an MRI and CT scan. The exclusion criteria were: (1). Revision surgery, (2). MRI and CT scan not available (3). Follow-up $<2$ years, (3). Associated with rotator cuff tear or superior labral anterior to posterior (SLAP) lesion, (4). Previous surgery around the shoulder, and (5) Patients with neuromuscular disorders or epilepsy.

\section{Preoperative preparation and Surgical procedure}

All the patients underwent routine preoperative imaging, including plain radiographs, MRI, and CT scan. MRI was used to evaluate the soft tissue status, including Bankart lesion, whereas CT scan was used to evaluate the bony status, including bony Bankart and Hill-Sach's lesion. To evaluate the glenoid bone loss, CT scan was compared with contralateral glenoid bone and measured using a surface area and glenoid ratio method. We considered $20 \%$ glenoid bone loss as critical bone loss and chose the type of surgery accordingly.

All the surgeries were performed under general anaesthesia (GA) with an additional dose of ultrasoundguided interscalene block.

\section{Arthroscopic Bankart repair}

The patient was placed on the lateral decubitus position, and all the anatomical landmarks, including border of acromion, clavicle, and coracoid process were drawn. Possible portal placements, including posterior portal (viewing portal) and anterosuperior and anteroinferior portals (working portals), were also drawn with a sterile marker. After the portal placement and diagnostic arthroscopy, anteroinferior glenoid rim and labrum were debrided and rasped to make sufficient bony bleeding [23]. At least 2 to 4 suture anchors (LUPINE (DePuy-Synthes)) were placed on the cartilaginous margin of the glenoid rim at 5:30 to 2 o'clock position. The glenoid labrum with a capsuloligamentous complex was then lifted up and tied with a sliding suture technique. If there was a significantly large and 'off-tract' Hill-Sach's lesion, then the Remplissage procedure was performed using a suture anchor.

\section{Latarjet procedure}

The patient was placed on a beach-chair position with a bolster on the interscapular region. We used a similar technique of the deltopectoral approach, as Edwards and Walch described for Latarjet procedure [24]. The coracoid graft fixation was performed with two $3.5 \mathrm{~mm}$ corticocancellous screws (CCS) or $4 \mathrm{~mm}$ malleolar screws with a washer, making sure that the screws are parallel to the glenoid surface. After adequate fixation was achieved, the coracoacromial (CA) ligament was routinely sutured to the capsule, subscapularis muscle was repaired, and the wound was closed in layers. However, care must be taken not to injure the coracoclavicular (CC) ligament. 


\section{Rehabilitation}

The shoulder immobilizer was used for 4 weeks. Pendulum exercise and passive forward flexion exercise was started immediately after surgery. However, active forward flexion and external rotation were permitted only after 6 weeks after surgery. Active range of motion (ROM) in all directions is advised at 3 months. We prescribed all the patients with similar rehabilitation protocol, and upon discharge from the hospital, all the patients were provided with a pamphlet for the instruction of rehabilitation.

\section{Clinical assessment}

Patients were scheduled for the follow-up visit at 6 weeks, 3 months, 6 months, 1 year, and yearly thereafter. Clinical outcomes were assessed using American Shoulder and Elbow Surgeons (ASES) score [25], Rowe score [26], and Quick Disabilities of the Arm, Shoulder and Hand (Quick DASH) score [27]. A self-constructed scale that consisted of satisfied and dissatisfied was used to measure the level of satisfaction. Any complications, including infection or redislocation, was recorded in every follow-up visit.

\section{Statistical analysis}

Collected data were analyzed using SPSS version 25. Chi-square test or Fisher Exact test was used for categorical variables and independent $t$-test for continuous variables. Categorical data were presented as number or percentage, and continuous data were presented as mean \pm standard deviation. A p-value of < 0.05 was considered a statistically significant difference.

\section{Result}

The demographic details of the patients are well illustrated in Table 1. There was no statistically significant difference existed between the Bankart group and Latarjet group. The patients' average age was 28 years in the Bankart group and 27 years in the Latarjet group $(p=0.401)$. Thirty-two $(78 \%)$ patients were male and the remaining 9 (22\%) patients were female in the Bankart group; whereas, 34 (85\%) patients were male and the remaining $6(15 \%)$ patients were female in the Latarjet group $(p=0.569)$. Similarly, 24 (58.5\%) patients had the right-sided dislocation and 17 (41.5\%) patients had left-sided dislocations in the Bankart group; whereas, $30(75 \%)$ patients had the right-sided dislocation and 10 $(25 \%)$ patients had left-sided dislocations in the Latarjet group $(p=0.158)$. The average number of dislocations was 9 episodes in the Bankart group and 10 episodes in the Latarjet group $(p=0.085)$. The average follow-up period of the Bankart group was 32 months and that of Latarjet group was 35 months $(p=0.061)$. 
Table 1. Demographic details of the patients (mean $\pm S D, n$ )

\begin{tabular}{|llll|}
\hline Parameters & Bankart Group $(\mathrm{n}=41)$ & Laterjet Group $(\mathrm{n}=40)$ & $\mathrm{p}$-value \\
\hline Age (years) & $28.76 \pm 10.35(16-48)$ & $27.10 \pm 7.00(18-68)$ & 0.401 \\
\hline Gender (M/F) & $32 / 9$ & $34 / 6$ & 0.569 \\
\hline Side (R/L) & $24 / 17$ & $30 / 10$ & 0.158 \\
\hline Number of dislocations & $9.02 \pm 4.30(3-19)$ & $10.86 \pm 4.20(5-23)$ & 0.085 \\
\hline Follow-up (months) & $32.66 \pm 7.49(24-48)$ & $35.83 \pm 7.47(24-53)$ & 0.061 \\
\hline SD: Standard deviation, n: Number of cases, M: Male, F: Female, R: Right side, L: Left side \\
\hline
\end{tabular}

The average ASES score was 85 points and 87 points in the Bankart group and Latarjet group, respectively $(p=0.388)$. Similarly, the average Rowe score was 84 points and 89 points in the Bankart group and Latarjet group, respectively $(p=0.211)$. Moreover, the average quick DASH score was 10 points and 9 points in the Bankart and Latarjet group, respectively $(p=0.713)$. The average external rotation was 83 degrees in the Bankart group and 85 degrees in the Latarjet group $(p=0.140)$. Thirty-five $(85.4 \%)$ patients were functionally satisfied and $6(14.6 \%)$ patients were dissatisfied in the Bankart group, whereas 37 (92.5\%) patients were functionally satisfied and $3(7.5 \%)$ patients were dissatisfied in the Latarjet group ( $p=0.482)$. Similarly, $100 \%$ of the patients were cosmetically satisfied in the Bankart group, whereas $32(80 \%)$ patients were cosmetically satisfied and $8(20 \%)$ patients were dissatisfied in the Latarjet group $(p=0.002)$ (Table 2$)$. 
Table 2. Clinical and functional outcomes of the patients (mean $\pm S D, n \%$ )

\begin{tabular}{|llll|}
\hline Parameters & Bankart Group $(\mathrm{n}=41)$ & Laterjet Group $(\mathrm{n}=40)$ & $\mathrm{p}$-value \\
\hline ASES score & $85.37 \pm 10.83(60-100)$ & $87.43 \pm 10.31(66-100)$ & 0.388 \\
\hline Rowe score & $84.15 \pm 19.55(20-100)$ & $89.23 \pm 16.24(35-100)$ & 0.211 \\
\hline Quick DASH score & $10.20 \pm 8.05(0-32)$ & $9.50 \pm 8.88(0-30)$ & 0.713 \\
\hline External rotation & $83.05 \pm 8.20(65-95)$ & $85.88 \pm 8.83(65-90)$ & 0.140 \\
\hline Functional satisfaction & & & 0.482 \\
\hline Satisfied & $35(85.4)$ & $37(92.5)$ & \\
\hline Dissatisfied & $6(14.6)$ & $3(7.5)$ & $0.002^{*}$ \\
\hline $\begin{array}{l}\text { Cosmetic satisfaction } \\
\text { Satisfied }\end{array}$ & $41(100)$ & $32(80)$ & \\
\hline $\begin{array}{l}\text { Dissatisfied } \\
\text { SD: Standard deviation, n: Number of cases, ASES: American Shoulder and Elbow Surgeons, DASH: } \\
\text { Disabilities of the Arm, Shoulder and Hand, *: Statistically significant difference exists between the } \\
\text { groups. }\end{array}$ & \\
\hline
\end{tabular}

There was a significantly higher operating cost for arthroscopic Bankart repair (average, USD 1059.46 \pm 191.30 (785-1488) compared to open Latarjet procedure (average, 622.52 $\pm 93.34(455-826)$ $(p<0.001)$.

\section{Recurrence and Complications}

Three patients had recurrence in the Bankart group, two of them underwent revision open Latarjet procedure, and another did not undergo the second surgery. No recurrence occurred in the Latarjet group. In the Latarjet group, 2 patients had superficial wound infection but relieved completely with a regular dressing and oral antibiotics. No other complications occurred in both groups.

\section{Discussion}

The most important findings of our study were that the differences in clinical and functional outcomes were not statistically significant in both the groups. The cosmetic satisfaction was $100 \%$ in the Bankart group, whereas it was only $80 \%$ in the Latarjet group. Three recurrence occurred in the Bankart group, while 2 superficial infections occurred only in the Latarjet group. The arthroscopic Bankart repair was more costly than the open Latarjet procedure. 
The Latarjet procedure for recurrent shoulder dislocation has been an established method for treating recurrent shoulder dislocation [28]. However, trends towards minimally invasive procedures have led the surgeons around the world to choose for the arthroscopic Bankart repair [7, 12, 29]. Thomazeau et al. [7] conducted a survey to find whether the shoulder surgeons preferred the Latarjet or arthroscopic Bankart repair. They found that irrespective of the status of the patients and glenoid bone loss, $72 \%$ of French shoulder surgeons would choose Latarjet procedure, while $90 \%$ of the shoulder surgeons from other countries would choose arthroscopic Bankart repair [7]. Some studies reported that the Bankart repair is more anatomic and would provide better shoulder ROM, stability, and minimal recurrence [12, 30-32]. In contrast, other reports suggested that the Latarjet procedure is superior to the Bankart repair, as it provides the triple-stabilizing effect, including anterior glenoid augmentation, capsular repair and sling effect, that significantly reduces the recurrence rate and a better return to preinjury status, especially in young and active individuals $[12,14,18,33]$. It is even effective in significant glenoid bone loss and revision for failed stabilization procedure [34].

An et al. [14] in their systematic review and meta-analysis concluded that the Latarjet procedure to be superior to the Bankart repair, offering better patient-reported outcomes, no restriction of external rotation, and provides more excellent stability without increasing complication. Our result showed that the open Latarjet procedure to produce better patient-reported outcomes compared to the arthroscopic Bankart procedure, and the external rotation was also not reduced in the Latarjet group. Our results add to the literature that the Latarjet procedure is a viable option with satisfactory clinical and functional outcomes $[12,33,18,17,6]$.

Patient-reported outcomes, following surgical stabilization procedure, are solely dependent upon the postoperative function, pain, and recurrence $[6,17,18]$. These factors also determine the functional satisfaction. As the surgery for recurrent shoulder dislocation is mainly indicated in young and active individuals, they require not only better function, but also, they have a tremendous aesthetic concern. This is one reason for choosing an arthroscopic Bankart repair by young and high-activity-demanding patients. Our result showed $100 \%$ of the patient's cosmetic satisfaction with the Bankart group; however, only $80 \%$ of patients were cosmetically satisfied in the Latarjet group. The dissatisfied people in the Latarjet group was mainly the young individuals who really cared about their cosmesis. However, proportion of functional satisfaction was higher $(92 \%)$ in the Latarjet group than the Bankart group $(85 \%)$. These results resemble the facts of previous findings that most of the patients are satisfied with the surgery; however, some are not. $[6,17,18,33]$. Another reason for choosing an arthroscopic procedure is the surgeon's discretion. Because of its fancy nature, surgeons insist on performing arthroscopic Bankart repair even in patients with significant glenoid bone loss or Hill-Sach's lesion.

According to An et al. [14], overall recurrence was $21 \%$ in the Bankart group and $11 \%$ in the Latarjet group. In our study, 3 (7.3\%) cases of recurrence occurred in the Bankart group. One redislocation occurred in a 24-year-old man during the gang fight, who had arthroscopic Bankart repair 27 months before. He had redislocation more than 5 times, and finally, he underwent open Latarjet procedure. Another recurrence also occurred in a male patient when he was playing volley-ball, who also underwent a 
successful Latarjet procedure. The last recurrence occurred in patients who had a sudden onset of seizure disorder for a different medical condition. This patient has not undergone any surgical procedure. No recurrence occurred in the Latarjet group. However, $41 \%$ of the patients in the Bankart group and 25\% of the patients in the Latarjet group had a positive apprehension sign. Such patients were not confident enough to go for overhead activities postoperatively. Various reasons could have played for the presence of persistent apprehension, including presence of hyper laxity, high activity level, lack of adequate postoperative rehabilitation, and inadequate fixation technique, etc. These results showed that there might be a possibility of recurrence in the future as the time passes because our follow-up period was only 2 years, or there could be more dislocations if the patients had performed preinjury activities, as most of our patients avoided such activities postoperatively.

Controversy exists regarding the cost-effectiveness of both the surgeries. Min et al. [35] found that arthroscopic Bankart repair to be more cost-effective as the actual cost of an open Latarjet procedure was $\$ 21398$ vs. $\$ 20,385$ for the arthroscopic Bankart group [35]. However, they still mentioned that the recurrence rate of arthroscopic Bankart procedure was higher as compared to the open Latarjet group, and they recommended open Latarjet procedure for a selected group of patients with high demand activities [35]. In contrast, Makhni et al. [36] found that the arthroscopic Bankart repair is more expensive than the open Latarjet procedure, leading the Latarjet procedure to be more dominant as it was more effective and less costly. The surgical cost in a developing country like Nepal is a huge burden for all people. The government of Nepal has recently implemented a basic medical insurance policy for the public of certain districts of the country [21]. That has made many patients to come to the hospital for definitive treatments for conditions like recurrent dislocation of the shoulder. Unfortunately, many patients do not have such basic medical insurance [22]. Even if they have, the implant costs are not covered. That puts a further burden on the patients. To have their surgery done, patients themselves or family members have to go abroad for earning, or even some have to sell their property [37, 38]. That's why many patients undergo an open Latarjet procedure that is cheaper than the arthroscopic Bankart procedure. In our study, the average cost for the Latarjet was significantly lower than the Bankart procedure, even if we used arthroscopic instruments multiple times that were supposed to be single-use only.

Despite a single experienced orthopaedic surgeon performed all the clinical examinations and not involved in the surgery to avoid the inter-observer bias, various limitations exist in our study. This study has all the biases that a retrospective and non-randomized study has. Despite a comparative study, our sample size was relatively small, and the follow-up period was not longer. Bigger sample size and longer follow-up period would have resulted in differently in terms of recurrence, as many of our patients have a positive apprehension sign and avoided preinjury activities. We prescribed a similar rehabilitation for all the patients; however, many patients did not attend the complete course, which might have directly affected the clinical outcomes of the patients. There might be an institutional bias as this study is from a single government hospital where mostly the economically poor patients come for the treatment.

\section{Conclusion}


Both procedures provided satisfactory clinical and functional outcomes. Cosmetic satisfaction was higher in the Bankart group, whereas functional satisfaction was higher in the Latarjet group. Despite being an expensive procedure, recurrence was also higher in the Bankart group compared to the Latarjet group. These results provide the fact that arthroscopic Bankart repair might be a fancy and minimally invasive procedure, Latarjet procedure should still be a priority in a developing country like Nepal, where financial cost is a huge burden.

\section{Abbreviation}

ASES: American Shoulder and Elbow Surgeons

CA: Coracoacromial

CC: Coracoclavicular

CCS: Corticocancellous screw

CT scan: Computed tomography scan

DASH: Disabilities of the Arm, Shoulder and Hand

GA: General anaesthesia

MRI: Magnetic resonance imaging

ROM: Range of motion

SLAP: superior labral anterior to posterior

SPSS: Statistical package for social sciences

\section{Declarations}

\section{Ethics approval and consent to participate}

Ethical clearance was obtained from the Ethical Review Committee of National Academy of Medical Sciences and written informed consent was obtained from participants before data collection

\section{Consent for publication}

Not applicable

\section{Availability of data and material}


The datasets supporting the conclusion of this article are included within the article. Upon genuine request, raw data can be provided by the corresponding author

\section{Competing interest}

The authors declare that they have no competing interests.

\section{Acknowledgement}

Not Applicable

\section{Funding}

None

\section{Author contribution}

All authors have read and approved the manuscript

BS: Supervision, Manuscript writing, Final draft approval

SR: Manuscript design, Manuscript writing, Final draft approval

NT: Data entry and analysis

LKS: Data entry, Data collection

JLS: Manuscript design, Language polishing

RPM: Supervision, Final draft approval

KK: Manuscript design, Final draft approval

\section{References}

1. Zacchilli MA, Owens BD. Epidemiology of Shoulder Dislocations Presenting to Emergency Departments in the United States: J Bone Jt Surg-Am Vol. 2010;92:542-9.

2. Sofu H. Recurrent anterior shoulder instability: Review of the literature and current concepts. World J Clin Cases. 2014;2:676.

3. Kralinger FS, Golser K, Wischatta R, Wambacher M, Sperner G. Predicting Recurrence after Primary Anterior Shoulder Dislocation. Am J Sports Med. 2002;30:116-20. 
4. Gibson K, Growse A, Korda L, Wray E, MacDermid JC. The effectiveness of rehabilitation for nonoperative management of shoulder instability: a systematic review. J Hand Ther Off J Am Soc Hand Ther. 2004;17:229-42.

5. Arciero RA, Wheeler JH, Ryan JB, McBride JT. Arthroscopic Bankart Repair Versus Nonoperative Treatment for Acute, Initial Anterior Shoulder Dislocations. Am J Sports Med. 1994;22:589-94.

6. Bessière C, Trojani C, Carles M, Mehta SS, Boileau P. The Open Latarjet Procedure Is More Reliable in Terms of Shoulder Stability Than Arthroscopic Bankart Repair. Clin Orthop Relat Res. 2014;472:2345-51.

7. Thomazeau H, Courage O, Barth J, Pélégri C, Charousset $C$, Lespagnol F, et al. Can we improve the indication for Bankart arthroscopic repair? A preliminary clinical study using the ISIS score. Orthop Traumatol Surg Res. 2010;96:S77-83.

8. Burkhart SS, De Beer JF. Traumatic glenohumeral bone defects and their relationship to failure of arthroscopic Bankart repairs. Arthrosc J Arthrosc Relat Surg. 2000;16:677-94.

9. Yamamoto N, Muraki T, Sperling JW, Steinmann SP, Cofield RH, Itoi E, et al. Stabilizing Mechanism in Bone-Grafting of a Large Glenoid Defect: J Bone Jt Surg-Am Vol. 2010;92:2059-66.

10. Shin S-J, Kim RG, Jeon YS, Kwon TH. Critical Value of Anterior Glenoid Bone Loss That Leads to Recurrent Glenohumeral Instability After Arthroscopic Bankart Repair. Am J Sports Med. 2017;45:1975-81.

11. Provencher MT, Ghodadra N, LeClere L, Solomon DJ, Romeo AA. Anatomic Osteochondral Glenoid Reconstruction for Recurrent Glenohumeral Instability With Glenoid Deficiency Using a Distal Tibia Allograft. Arthrosc J Arthrosc Relat Surg. 2009;25:446-52.

12. Blonna D, Bellato E, Caranzano F, Assom M, Rossi R, Castoldi F. Arthroscopic Bankart Repair Versus Open Bristow-Latarjet for Shoulder Instability: A Matched-Pair Multicenter Study Focused on Return to Sport. Am J Sports Med. 2016;44:3198-205.

13. Camus D, Domos P, Berard E, Toulemonde J, Mansat P, Bonnevialle N. Isolated arthroscopic Bankart repair vs. Bankart repair with "remplissage" for anterior shoulder instability with engaging Hill-Sachs lesion: A meta-analysis. Orthop Traumatol Surg Res OTSR. 2018;104:803-9.

14. An VVG, Sivakumar BS, Phan K, Trantalis J. A systematic review and meta-analysis of clinical and patient-reported outcomes following two procedures for recurrent traumatic anterior instability of the shoulder: Latarjet procedure vs. Bankart repair. J Shoulder Elbow Surg. 2016;25:853-63.

15. Zhu Y-M, Lu Y, Zhang J, Shen J-W, Jiang C-Y. Arthroscopic Bankart repair combined with remplissage technique for the treatment of anterior shoulder instability with engaging Hill-Sachs lesion: a report of 49 cases with a minimum 2-year follow-up. Am J Sports Med. 2011;39:1640-7.

16. Purchase RJ, Wolf EM, Hobgood ER, Pollock ME, Smalley CC. Hill-Sachs "Remplissage”: An Arthroscopic Solution for the Engaging Hill-Sachs Lesion. Arthrosc J Arthrosc Relat Surg. 2008;24:723-6.

17. Xu Y, Wu K, Ma Q, Zhang L, Zhang Y, Xu W, et al. Comparison of clinical and patient-reported outcomes of three procedures for recurrent anterior shoulder instability: arthroscopic Bankart repair, 
capsular shift, and open Latarjet. J Orthop Surg. 2019;14:326.

18. Hovelius L, Vikerfors O, Olofsson A, Svensson O, Rahme H. Bristow-Latarjet and Bankart: a comparative study of shoulder stabilization in 185 shoulders during a seventeen-year follow-up. $J$ Shoulder Elbow Surg. 2011;20:1095-101.

19. Matthes G, Horvath V, Seifert J, Ptok H, Stengel D, Schmucker U, et al. Oldie but Goldie: BristowLatarjet Procedure for Anterior Shoulder Instability. J Orthop Surg. 2007;15:4-8.

20. Young DC, Rockwood CA. Complications of a failed Bristow procedure and their management. J Bone Jt Surg-Am Vol. 1991;70-A.

21. Health Insurance Board. https://hib.gov.np/en/. 2020.

22. Ghimire R. Community Based Health Insurance Practices in Nepal. 2013;2:11.

23. Cole BJ, Romeo AA. Arthroscopic Shoulder Stabilization With Suture Anchors: Technique, Technology, and Pitfalls: Clin Orthop. 2001;390:17-30.

24. Edwards TB, Walch G. The Latarjet Procedure for Recurrent Anterior Shoulder Instability: Rationale and Technique. Oper Tech Sports Med. 2012;20:57-64.

25. Michener LA, McClure PW, Sennett BJ. American Shoulder and Elbow Surgeons Standardized Shoulder Assessment Form, patient self-report section: reliability, validity, and responsiveness. J Shoulder Elbow Surg. 2002;11:587-94.

26. Rowe CR, Patel D, Southmayd WW. The Bankart procedure: a long-term end-result study. J Bone Joint Surg Am. 1978;60:1-16.

27. Hudak PL, Amadio PC, Bombardier C. Development of an upper extremity outcome measure: the DASH (disabilities of the arm, shoulder and hand) [corrected]. The Upper Extremity Collaborative Group (UECG). Am J Ind Med. 1996;29:602-8.

28. Latarjet M. [Treatment of recurrent dislocation of the shoulder]. Lyon Chir. 1954;49:994-7.

29. Boileau P, Richou J, Lisai A, Chuinard C, Bicknell RT. The role of arthroscopy in revision of failed open anterior stabilization of the shoulder. Arthrosc J Arthrosc Relat Surg Off Publ Arthrosc Assoc N Am Int Arthrosc Assoc. 2009;25:1075-84.

30. Bokshan SL, DeFroda SF, Owens BD. Comparison of 30-Day Morbidity and Mortality After Arthroscopic Bankart, Open Bankart, and Latarjet-Bristow Procedures: A Review of 2864 Cases. Orthop J Sports Med. 2017;5:232596711771316.

31. Chen L, Xu Z, Peng J, Xing F, Wang H, Xiang Z. Effectiveness and safety of arthroscopic versus open Bankart repair for recurrent anterior shoulder dislocation: a meta-analysis of clinical trial data. Arch Orthop Trauma Surg. 2015;135:529-38.

32. Cole BJ, Warner JJP. Arthroscopic versus open Bankart repair for traumatic anterior shoulder instability. Clin Sports Med. 2000;19:19-48.

33. Hovelius LK, Sandström BC, Rösmark DL, Saebö M, Sundgren KH, Malmqvist BG. Long-term results with the Bankart and Bristow-Latarjet procedures: Recurrent shoulder instability and arthropathy. J Shoulder Elbow Surg. 2001;10:445-52. 
34. Flinkkilä T, Sirniö K. Open Latarjet procedure for failed arthroscopic Bankart repair. Orthop Traumatol Surg Res. 2015;101:35-8.

35. Min K, Fedorka C, Solberg MJ, Shaha SH, Higgins LD. The cost-effectiveness of the arthroscopic Bankart versus open Latarjet in the treatment of primary shoulder instability. J Shoulder Elbow Surg. 2018;27:S2-9.

36. Makhni EC, Lamba N, Swart E, Steinhaus ME, Ahmad CS, Romeo AA, et al. Revision Arthroscopic Repair Versus Latarjet Procedure in Patients With Recurrent Instability After Initial Repair Attempt: A Cost-Effectiveness Model. Arthrosc J Arthrosc Relat Surg. 2016;32:1764-70.

37. Kunwar LS. Cross-border migration process of Nepalese people to India. Nepal Popul J. 2018;18:8190.

38. Malla B, Rosenbaum MS. Understanding Nepalese Labor Migration to Gulf Countries. J Poverty. 2017;21:411-33. 